\title{
Investigating the factor structure of a translated recovery-orientation instrument in inpatient treatment for substance use disorder
}

Dagny Adriaenssen Johannessen ${ }^{1,2^{*}}$ (D), Amy Østertun Geirdal ${ }^{2}$ and Trond Nordfjærn ${ }^{3,4}$

\begin{abstract}
Background: Recovery has been outlined as a process of change through which involvement and empowerment enables individuals to reach their goals and aspirations. Recovery self-assessment (RSA) is an instrument that has been acknowledged as an applicable measure of recovery-orientation in services for people with mental health problems or substance use disorder (SUD). This study aimed to translate RSA from US English to Norwegian and to investigate the factor structure of the translated version (RSA-N).

Methods: A translate/back-translate procedure was used. Confirmatory factor analysis (CFA) was applied to investigate the factor structure of RSA-N in a sample of clinicians $(n=407)$ working in inpatient SUD treatment facilities.

Results: The results suggested that the hypothesised five-factor structure originally obtained by the developers showed an inadequate fit with the current data sample. RSA-N was modified and restructured by removing twelve misfitting items and combining factors with high covariance using data from one subsample. The alternative threefactor structure yielded an acceptable fit for the data from a second subsample. Acceptable alpha coefficients, suggesting good internal consistency, supported the adequacy of the three-factor structure.

Conclusions: Results from the present study are in line with previous findings, which have failed to replicate the hypothesised five-factor structure without modifications. Knowledge about the degree to which SUD services are recovery-oriented may contribute to SUD services' pursuit of establishing an inpatient treatment environment that fosters change and development of inpatients. The present study's findings imply RSA-N's potential as an instrument to assess recovery-orientation in inpatient SUD treatment.
\end{abstract}

Keywords: Confirmatory factor analysis, Recovery-orientation, Recovery self-assessment, Substance use disorder, Translate/back-translate

\footnotetext{
* Correspondence: dagnyaj@live.com

'Blue Cross East, Oslo, Norway

${ }^{2}$ Department of Social Work, Child Welfare and Social Policy, OsloMet - Oslo

Metropolitan University, Oslo, Norway

Full list of author information is available at the end of the article
}

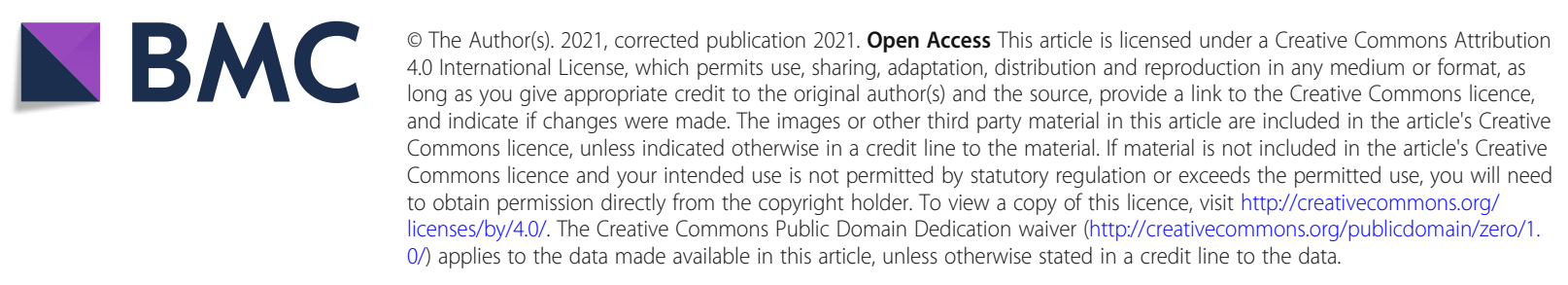




\section{Background}

In the course of time, recovery-orientation has been established as part of public services for people with substance use disorder (SUD) or mental health problems [1-4]. Recovery communities are occupied with how people who experience negative consequences of substance use or poor mental health are perceived and approached. Recovery has been outlined as a process of change where involvement and empowerment facilitate the necessary autonomy, self-perception and belonging to reach individually defined goals and to live a life that the individual finds meaningful [5-7]. Recovery may thus be regarded as a goal or a process, as well as a measure to establish an environment that fosters change and development $[5,6]$. Recovery as a goal implies leading a meaningful life as defined by the individual and as a process, recovery involves changes in life domains that are affected by the negative consequences of substance use or mental health problems [8]. Recovery as a measure refers to a framework that promotes the process and the goal of recovery, often implemented by services in their pursuit of becoming recovery-oriented services [7].

Several instruments have been developed to measure the degree to which SUD and mental health services are recovery-oriented [3, 9]. One is Recovery self-assessment (RSA: [10]), a validated self-report instrument with satisfactory internal consistency, which has been an applicable measure of recovery-orientation and acknowledged as such [3]. RSA was developed in the United States (US) to measure recovery-orientation in community, outpatient or inpatient services. Separate versions of RSA are available for different target groups, comprising users, family members, service providers and managers. RSA can be used in one or several of these target groups to assess recovery-orientation in services or for research purposes.

RSA for users has previously been translated from US English to Chinese [11], Swedish [12], Malaysian [13] and Hong Kong Chinese [14], in which all have been tested for validity among people attending community mental health services. More recently, RSA for users has been translated to Brazilian Portuguese and culturally adapted to community mental health services in Brazil but has not yet been psychometrically tested [15].

The providers' version of RSA has previously been translated from US English to German [16] and tested for internal consistency among the staff of a psychiatric inpatient hospital [17]. Validity studies have revealed diverging psychometric properties of the providers' version of RSA in psychiatric inpatient hospital settings. Salyers, Tsai and Stultz [18] found that RSA had good internal consistency and stable test-retest reliability. In contrast, Thege, Ham and Ball [19] failed to confirm the hypothesised factor structure in their data sample.
The providers' version of RSA has been used to explore associations between mental health clinicians' perception of the degree to which services are recoveryoriented, on one hand, and different factors, such as job satisfaction, stigmatising attitudes, therapeutic alliance with service users, and recovery outcome among users, on the other hand [20-24]. However, RSA has hardly been used to study recovery-orientation in specialised SUD services. During the development of RSA, staff from both mental health and SUD services participated, but the results were aggregated [10]. To the best knowledge of this paper's authors, no studies have used RSA to assess clinicians' perception of recovery-orientation specifically in SUD services or have investigated its validity in a population of clinicians working in specialised inpatient SUD treatment facilities.

Specialised treatment for SUD may be considered a planned turning point where basic needs are met and development and change are initiated [25]. Inpatient SUD treatment has been recognised as particularly important for individuals with SUD who have multiple psychosocial challenges and struggle in managing their everyday life [26-28]. Recovery-orientation has been established as an expedient way to organise and provide services for individuals who undergo extensive changes [29-31]. RSA captures several of the enabling factors in the vast change process that is often involved in recovering from SUD, including social support, belonging, motivation, involvement and predictability in terms of basic needs and rate of change [32-38].

People with SUD are more often targeted with stigmatising attitudes compared to people with mental health problems [39, 40]. Stigmatising attitudes also appear among clinicians working in the treatment of mental health problems or SUD [41]. A recovery-oriented framework includes measures to counter common negative beliefs about people with SUD or mental health problems. These measures involve addressing stigmatising descriptions of people with SUD or informing the general public about SUD and its recovery potential. Such measures may contribute to reduced stigma and discrimination, both among clinicians $[42,43]$ and the general public $[39,44]$; A wider assessment of the recovery-orientation in SUD services is therefore significant.

Few instruments are suitable for assessing the degree of recovery-orientation in SUD services, as perceived by clinicians, and with qualities similar to those of RSA. RSA may be a useful tool in exploring recoveryorientation in inpatient SUD treatment.

This study had two aims: 1) to formally translate the providers' version of RSA from US English to Norwegian and 2) to investigate the factor structure of the translated version among clinicians working in inpatient SUD treatment facilities. 


\section{Materials and methods}

\section{Recovery Self-Assessment (RSA)}

RSA includes 36 items, which are rated on a 5-point Likert scale (from 1 = "strongly disagree" to 5 = "strongly agree"), with two additional options (N/A = "not applicable" and $\mathrm{D} / \mathrm{K}=$ "don't know"). The items are divided into five subscales that provide information about the services' ability to promote service users' recovery. The subscales are hypothesised to measure the extent to which service users' Life goals, Involvement, and Choice are promoted by the service staff. The instrument also covers the degree to which the services offer a Diversity of treatment options and are Individually-tailored services. The instrument provides individual scores for each of the five subscales, as well as a total score. Scores on the individual subscales yield information about potential areas of improvement to establish a recoveryoriented environment in the treatment facility or service. High scores suggest that the services are recoveryoriented, while low scores indicate the opposite [10].

\section{Translation procedure}

RSA was translated according to a translate/back-translate procedure and guidelines for translation [45] (see Table 1).

In the preparation phase, the five RSA subscales were thoroughly reviewed to evaluate if they captured the factors that have previously been outlined as enablers in the vast change process involved in recovering from SUD. The RSA developers do not require stakeholders to obtain permission to use the instrument (i.e., no copyright) $[3,46]$. However, the originator was informed about the research project and the plans for translation before the procedure started.
RSA was forward-translated from US English to Norwegian by two of the authors (DAJ and AØG), who have clinical and research experience in mental health and SUD and are native Norwegian speakers. The forward-translation was adapted to the Norwegian inpatient SUD treatment setting in terms of language, culture and organisation of health services. The translated version was then back-translated by a professional translator with no knowledge of the original instrument.

The back-translation was reviewed and compared with the original instrument. The authors discussed the differences between the original and the back-translated versions in terms of conceptual correspondence and adjusted the forward-translated version accordingly.

The adjusted forward-translated version was first sent to clinicians $(n=6)$ working in a specialised outpatient SUD treatment programme. They were asked to complete the instrument and comment on the wording, concepts, understandability and relevance of the included items. Their responses were reviewed, and the translated version was adjusted according to their feedback.

The adjusted version was then sent to representatives $(n=4)$ of non-governmental organisations (NGO) in the SUD field for further assessment. They were asked to comment on the language, concepts, understandability and relevance of the instrument. They were also instructed to proofread the instrument. The adjusted translation was then modified according to their feedback, and the instrument was established as Recovery Self-Assessment - Norwegian (RSA-N). The translation procedure took place from January to July 2020.

Table 1 Translation procedure according to Wild and colleagues' [45] guidelines

\begin{tabular}{|c|c|}
\hline Procedure & Description \\
\hline Preparations & $\begin{array}{l}\text { The literature was searched for Norwegian translations and the originator of RSA was informed about the research } \\
\text { project }\end{array}$ \\
\hline Forward translation & $\begin{array}{l}\text { RSA was translated from US English to Norwegian by two native Norwegian speakers with clinical and research } \\
\text { experience in mental health and SUD }\end{array}$ \\
\hline Revise & $\begin{array}{l}\text { The forward-translation was adapted to the Norwegian inpatient SUD treatment setting in terms of language, culture } \\
\text { and organisation of health services }\end{array}$ \\
\hline Back translation & $\begin{array}{l}\text { The forward-translation was back-translated from Norwegian to US English by a professional translator with no know- } \\
\text { ledge of the original instrument }\end{array}$ \\
\hline Review & The back-translation was reviewed and compared to the original instrument \\
\hline Harmonization & $\begin{array}{l}\text { The authors discussed differences in the original- and back translated versions in terms of conceptual correspondence } \\
\text { and adjusted the forward-translated version }\end{array}$ \\
\hline External assessment & $\begin{array}{l}\text { Clinicians completed the instrument and commented on wording, concepts, understandability and relevance of the } \\
\text { included items }\end{array}$ \\
\hline Adjustment & The translated version was adjusted according to feedback from the clinicians \\
\hline $\begin{array}{l}\text { External assessment and } \\
\text { proofreading }\end{array}$ & $\begin{array}{l}\text { NGO-representatives commented on language, concepts, understandability, relevance of the instrument and proofread } \\
\text { the instrument }\end{array}$ \\
\hline Adjustment and finalisation & The adjusted translation was finalised according to feedback from the NGO-representatives \\
\hline
\end{tabular}




\section{Setting and participants Setting}

Regarding this study's second aim, the factor structure was investigated in Norway, where specialized outpatient and inpatient SUD treatments are organised under four regional health enterprises (Northern Norway Regional Health Authority, Central Norway Regional Health Authority, Western Norway Regional Health Authority, and Southern and Eastern Norway Regional Health Authority). These health enterprises award private organisations with contracts to provide specialised SUD treatment. The contractual agreement ensures that the private providers adhere to formal requirements. The expenses for inpatient SUD treatment are covered with public funds, and people who undergo outpatient treatment pay a small deductible.

In line with those of other western countries, Norway's specialised inpatient SUD treatment is interdisciplinary and consists of psychological, social and medical interventions and measures. Administered in the treatment facility where the patients reside, inpatient SUD treatment normally comprises individual, environmental and group therapies [47-49]. To adhere to the ideal of interdisciplinarity in inpatient SUD treatment facilities, psychologists, social workers, nurses, medical doctors and psychiatrists are normally employed.

Inpatient SUD treatment facilities in Norway adhere to various therapeutic orientations. Some provide treatment that originates from the psychodynamic tradition, such as mentalisation-based therapy. Others provide treatment from the recovery tradition. Among these are Hierarchical Therapeutic Communities (CTC) and inpatient twelve-step programmes. Several of the facilities are treatment collectives. These have a democratic structure, like Democratic Therapeutic Communities (DTC), but originated partly through inspiration from pedagogical collectives for adolescents with behavioural difficulties in Sweden and the Soviet Union. Lastly, other inpatient SUD treatment facilities adhere to cognitive behavioural therapy or describe their therapeutic orientation as eclectic.

\section{Participants}

The study protocol was independently reviewed and approved by the Norwegian Centre for Research Data (NSD; reference number 883511). The participants received written information and gave their consent by answering the first item in the questionnaire: "I give my consent to participate in the study and to my answers being stored in Sensitive Data Services (TSD) and used for the purpose of research."

Fifty-four eligible treatment facilities were invited to participate in the study. Among these, 50 facilities responded to the invitation, and a contact person from each facility provided clinical staff members with a link to a self-report questionnaire via e-mail. Additionally, potential respondents received one or two reminders to answer the questionnaire. Ideally, an equal number of participants from all facilities should be included. However, to attain a sufficient number of participants, all clinical staff members at each facility were invited. The participating facilities employed from 10 to 50 clinicians in total $($ mean $=15$, median $=16)$. More participants therefore contributed from larger facilities than from smaller facilities. In total, 426 of the 933 (response rate = $46 \%$ ) invited clinicians completed the questionnaire; $96 \%(n=407)$ of these respondents reported that they worked directly (i.e., clinically) with the inpatients in the treatment facility. The respondents who did not work clinically $(n=19)$ were excluded from the study. The participants comprised clinicians working in long-term ( $\geq 6$ months) inpatient SUD treatment facilities in Norway. The data were collected from August to October 2020 .

\section{Investigating the factor structure Measures}

The questionnaire consisted of RSA-N and six items that collected demographic information, including age, gender, number of years spent working in the SUD field, number of years spent working in an SUD treatment facility, and job title. One of the items from the original RSA concerned accessibility by collecting information about the place where the services were provided. During inpatient treatment, the inpatient's reside in the treatment facility; thus, the item was removed, as suggested by Campbell-Orde, Chamberlin, Carpenter and Stephen Leff [46]. The RSA-N therefore consisted of 35 items, which were formulated as statements about the treatment programme. The questionnaire was completed by assessing the 35 RSA-N items on a 5-point Likert scale (for the scoring options, see above), as well as the six demographic items, which took approximately 15 min to complete.

The total RSA-N score was obtained by computing the mean of all 35 items (ranging from 1 to 5). The individual subscale scores were obtained by computing the mean of the items included in each subscale (ranging from 1 to 5), as suggested by the originators [10].

\section{Analytical strategy}

Confirmatory factor analysis (CFA) is normally applied when the structure of a measurement instrument, such as RSA, has been established through assessing which items are pooled together in a latent factor (i.e., a subscale) [50]. CFA was therefore conducted to investigate whether the hypothesised five-factor structure of the original RSA, as described by O'Connell, Tondora, Croog, 
Evans and Davidson [10], coincided with the factor structure of RSA-N in the current data sample. Due to poor fit indices, the sample was randomly divided into two equally sized subsamples, which provided an opportunity to modify RSA-N with a sample that differed from the one used to test the validity of the modified RSA-N (alternative model).

Principal component analysis (PCA) and modification indices in CFA may be used to remove items and respecify the factor structure of an instrument [50]. Therefore, the data from subsample 1 were used to identify cross-loading items, items with weak loadings (i.e., misfitting items) and covariance between hypothesised factors using PCA and modification indices in CFA. The data from subsample 2 were used to test the validity of the alternative model.

The chi-square test and approximate fit indices designate how well the data fit the hypothesised factor structure (measurement model) by using information about common and unique variances of the indicators (items and latent factors) obtained with the observed data. The fit indices estimated the model fit by placing the observed data on a range from a poor model (baseline model), with no information about variance, to a good model (saturated model), with all the information about variance.

The chi-square $\left(x^{2}\right)$ test, root mean square error of approximation (RMSEA) with $90 \%$ confidence interval (90\% CI) and comparative fit index (CFI) were used to evaluate the fit of the hypothesised factor structure in the current data sample. In the chi-square test, small differences between the data and the hypothesised model are preferred, and $p$-values above 0.05 may indicate a good fit. The chi-square test is sensitive to both small and large sample sizes [51]. The two approximate fit indices, RMSEA and CFI, were therefore emphasised in evaluating the adequacy of the hypothesised five-factor structure for the current data. A low RMSEA is desirable, and values above 0.08 and an upper confidence interval value above 0.1 may indicate a poor fit [52]. A high CFI is desirable, and the fit may be acceptable with values close to 0.90 [53]. Internal consistency was considered using Cronbach's alpha coefficients $(\alpha)$, which estimate the adequacy of pooling designated items together to measure a latent construct or a subscale. Alpha coefficients with values of 0.7 and above indicate acceptable internal consistency for the data [51].

Descriptive statistics were used to examine the respondents' personal and professional characteristics, as well as patterns of system-missing values (additional response options N/A and D/K). Mean imputation, as described by Christophersen [54] (the item mean value plus the subscale mean value divided by two), was used on items with $90 \%$ or more valid responses [55]. All analyses were conducted both with and without mean imputation, providing the same results in terms of factor structure and misfitting items.

Pattern analyses of system-missing values were performed using IBM SPSS Statistics Version 27, while jamovi version 1.2.27 was used for all other analyses [56].

\section{Results}

\section{Sample characteristics}

The respondents' personal and professional characteristics are presented in Table 2. The participants' mean age was 44.7 years, and $68 \%$ were women. The mean time spent working in the SUD field was 9.96 years, and the mean time spent working in an inpatient SUD treatment facility was 7.7 years. Among the participants, 35\% were medical staff members, $16 \%$ served as social workers, $11 \%$ worked as psychologists or therapists, and $38 \%$ were registered as other staff, including peer specialists, environmental therapists, financial counsellors and job

Table 2 Respondents' personal and professional characteristics

\begin{tabular}{|c|c|c|}
\hline & $\mathrm{n}(\%)$ & $\begin{array}{l}\text { Total } n=407 \\
\text { mean (SD) }\end{array}$ \\
\hline \multicolumn{3}{|l|}{ Gender } \\
\hline Women & $275(68)$ & \\
\hline Men & $132(32)$ & \\
\hline Age & & $44.7(10.6)$ \\
\hline $20-30$ & $44(12)$ & \\
\hline $31-40$ & $99(26)$ & \\
\hline $41-50$ & $121(31)$ & \\
\hline $51-60$ & $97(25)$ & \\
\hline$>60$ & $23(6)$ & \\
\hline \multicolumn{3}{|l|}{ Job title } \\
\hline Medical staff & $145(35)$ & \\
\hline Social worker & $64(16)$ & \\
\hline Psychologist and therapist & $44(11)$ & \\
\hline Other staff & $154(38)$ & \\
\hline Years spent working in the SUD field & & $9.96(7.38)$ \\
\hline$<5$ & $120(30)$ & \\
\hline $5-10$ & $131(32)$ & \\
\hline $11-15$ & $69(17)$ & \\
\hline $16-20$ & $50(12)$ & \\
\hline$>20$ & $37(9)$ & \\
\hline Years spent working with SUD treatment & & $7.70(6.45)$ \\
\hline$<5$ & $164(40)$ & \\
\hline $5-10$ & $139(34)$ & \\
\hline $11-15$ & $52(13)$ & \\
\hline $16-20$ & $33(8)$ & \\
\hline$>20$ & $19(5)$ & \\
\hline
\end{tabular}


counsellors. Four items (4, 27, 12 and 15) had more than $10 \%$ system-missing values, with valid responses ranging from $n=319$ to $n=378$.

CFA was conducted to investigate how well the fivefactor structure, originally obtained by O'Connell, Tondora, Croog, Evans and Davidson [10], would fit the current data. The RMSEA (90\% CI) values (0.052 (0.048-0.056)) suggested an adequate fit, while the CFI (0.083) indicated a poor fit (chi-square test: $\mathrm{X}^{2}(\mathrm{df})=$ 1155.982 (550), $p<0.01$ ). Additionally, the factor covariance between Life Goals and Choice was high (0.95), as was the factor covariance between the two hypothesised factors: Diversity of treatment options and Individually-tailored services (0.94). High covariation between factors indicates related or overlapping factors, which is fairly common in multidimensional psychometric instruments. However, high values normally imply that the model has poor discriminant validity and should be respecified [50].

Due to these results, the study group was randomly divided into two equal subsamples: subsample $1(n=203)$ and subsample $2(n=204)$. RSA-N was modified and respecified using PCA and modification indices in CFA with the data from subsample 1 . The modification resulted in an alternative model, which was tested with CFA using the data from subsample 2.

\section{Modifying RSA-N}

As some correlations between the components were expected, oblique rotation with Promax was applied during the PCA. Bartlett's test of Sphericity $\left(X^{2}(\mathrm{df})=1496.080\right.$ (595), $p<0.01)$ and Kaiser-Meyer-Olkin (KMO) of Sampling Adequacy (MSA $=0.78$ ) implied that the data were suitable for PCA [57].
The items that did not load with values over 0.3 on a component in the PCA were removed, as were the cross-loading items with values over 0.4 [50]. One item (29, see Table 3 ) did not load over 0.3 , and none of the items cross-loaded.

Ten items $(1,4,6,12,13,16,19,20,26$ and 32; see Table 3$)$ with weak loading $(<0.4)$ on the designated factor or high loadings on several factors were identified and removed one by one, using modification indices in CFA [50].

\section{Respecifying the factor structure}

The original factor structure was respecified by investigating common and unique variances between the items and the latent factors in CFA. The covariance between two pairs of the hypothesised factors - Life goals and Choice, and Diversity of treatment options and Individually-tailored services - remained high.

Life goals consist of items addressing staff members' role in helping inpatients outline and achieve their individually defined goals, such as "Staff actively assist patients with the development of career and life goals that go beyond symptom management and stabilization." Choice includes items that gather information about the extent to which staff members use measures to influence inpatients' choices connected to defining their individual goals, such as "Staff at this agency listen to and follow the choices and preferences of the patients."

Diversity of treatment options contains items that collect information about the degree to which various treatment options are offered and whether the treatment programme is varied in terms of inpatients' individual needs, such as "Groups, meetings and other activities can be scheduled in the evenings or on weekends so as

Table 3 Removed items

\begin{tabular}{|c|c|}
\hline Item & Statement \\
\hline 1. & $\begin{array}{l}\text { Helping the patients build connections with their neighbourhoods and communities is one of the primary activities in } \\
\text { which staff at this agency are involved }\end{array}$ \\
\hline 3. & The patients have access to all their treatment records \\
\hline 4. & This agency provides education to community employers about employing people with mental illness and/or addictions \\
\hline 6. & The patients can choose and change, if desired, the therapist, psychiatrist, or other service provider with whom they work \\
\hline 12. & This agency provides structured educational activities to the community about mental illness and addictions \\
\hline 13. & Agency staff do not use threats, bribes, or other forms of coercion to influence the patient's behaviour or choices \\
\hline 16. & Staff are knowledgeable about special interest groups and activities in the community \\
\hline 19. & $\begin{array}{l}\text { This agency provides a variety of treatment options (i.e., individual, group, peer support, holistic healing, alternative } \\
\text { treatments, medical) from which the patients may choose }\end{array}$ \\
\hline 20. & The achievement of goals by patients and staff are formally acknowledged and celebrated by the agency \\
\hline 26. & Agency staff are diverse in terms of culture, ethnicity, lifestyle, and interests \\
\hline 29. & Staff routinely assist patients in the pursuit of educational and/or employment goals \\
\hline 32. & $\begin{array}{l}\text { This agency provides formal opportunities for patients, family members, service providers, and administrators to learn } \\
\text { about recovery }\end{array}$ \\
\hline
\end{tabular}


not to conflict with other recovery-oriented activities, such as employment or school." Individually-tailored services comprise items that gather information about the extent to which the treatment programme is customised to meet inpatients' individual needs, such as "This agency offers specific services and programmes for individuals with different cultures, life experiences, interests and needs."

The two factors - Life goals and Choice - were combined and established as Goals and choice. Likewise, Individually-tailored services and Diversity of treatment options were merged and established as Individually tailored and varied (for the alternative three-factor solution, see Additional file 1). Model fit indices for the alternative three-factor model showed improved results. RMSEA (90\% CI) (0.055 (0.043-0.064)) and CFI (0.87) indicated an acceptable fit (chi-square test: $\mathrm{X}^{2}(\mathrm{df})=$ 394.5516 (249), $p<0.01$ ).

\section{Testing the alternative model}

The alternative three-factor structure was tested using CFA with data from subsample 2 . The results are presented in Fig. 1. One item (3, see Table 3) was removed due to weak loading $(<0.4)$.

Model fit indices indicated that the alternative factor structure was applicable, with RMSEA (90\% CI) $(0.059$ (0.049-0.069)) and CFI (0.89) both indicating an acceptable fit (chi-square test: $\mathrm{X}^{2}(\mathrm{df})=388.1141$ (227), $p<$ 0.01).

The factor loadings (standardised) ranged from 0.43 to 0.81 and were significant $(p<0.05)$. The covariance (standardised) between the factors ranged from 0.51 to 0.85 and were significant $(p<0.05)$. Reliability analyses indicate good internal consistency for the three factors Goals and choice $(\alpha=0.82)$, Involvement $(\alpha=0.74)$ and Individually tailored and varied $(\alpha=0.75)$ - and for the overall RSA-N instrument $(\alpha=0.88)$.

\section{Discussion}

The main results of the present study are that the fivefactor structure originally obtained by O'Connell, Tondora, Croog, Evans and Davidson [10] has an inadequate fit with the current data from the SUD sector. Twelve misfitting items were removed during the modification, and factors with high covariance were combined. An alternative three-factor model is proposed for RSA-N, comprising the subscales Goals and choice, Involvement and Individually tailored and varied. Approximate fit indices for the alternative model were found to be acceptable, as was the internal consistency according to alpha coefficients.

This present study's main findings coincide with prior validity studies with translated versions of RSA, which have found acceptable internal consistency for the subscales included in the users' version of RSA [11-13, 17] and fair to good internal consistency for the subscales included in the providers' version $[17,18]$.

In line with the present study's results, previous studies of the factor structure have failed to replicate the hypothesised five-factor structure without modifications. Based on the CFA results, Tan and Fernandez [13] concluded that the five-factor structure could be replicated by allowing the error terms of eight items to covary. Tan and Fernandez [13] study is the only one that has successfully replicated the original five-factor structure using a translated version of RSA. Furthermore, based on their results from a Rasch analysis, the authors suggested that the Life Goals subscale should be split into two, which they recommended for further use in the Chinese version of RSA [11]. Based on exploratory factor analysis with parallel analysis, Ye, Pan, Wong and Bola [14] suggested that three factors could be extracted, and Lodge, Kuhn, Earley and Manser [58] reported that a one-factor solution provided the best fit for the data. Although Ye, Pan, Wong and Bola [14] found a threefactor solution with their data sample, due to their research aims, they decided to use a one-factor solution and therefore did not provide supplementary information about the three-factor solution. Lastly, Thege, Ham and Ball [19] tested the psychometric properties of the original RSA with CFA. Based on unacceptable fit indices and high covariance between several of the latent factors, they concluded that the original five-factor structure had an inadequate fit in a sample of staff members working with inpatient treatment for severe mental illness.

None of these prior studies have tested the hypothesised factor structure in a sample of clinicians working with inpatient SUD treatment. Furthermore, we found no previous studies that explored the adequacy of using RSA to assess recovery-orientation in SUD services in general or inpatient SUD treatment in particular since the RSA was developed.

Several factors may explain the issue with replicating the original five-factor structure shown in this and previous validation studies of RSA. First, there are variations in how people with mental health problems are approached compared to people with SUD. The most common notion of recovery applied in the European SUD field today is derived from movements such as the twelve-step tradition and sobriety movements, where eliminating the addictive substance (i.e., the symptom) represents a core value [59-61]. In the mental health field, however, the prevailing notion of recovery developed as part of a civil rights movement. The movement emerged during the 1960s and consisted of people with mental health problems who advocated their right and opportunity to take part in society on equal terms to 


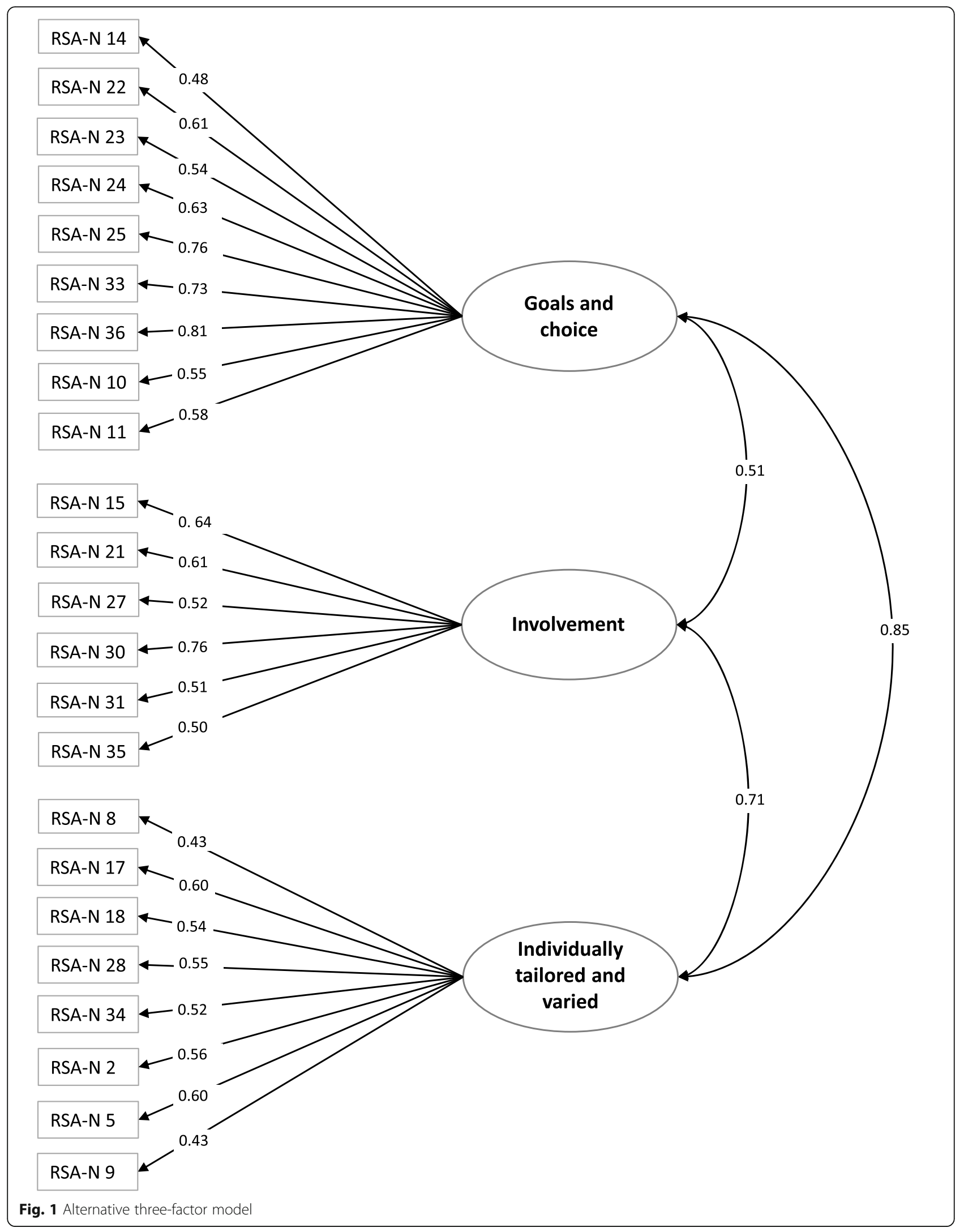


other citizens, regardless of their symptoms [2]. The main differences in treatment approaches between these recovery orientations may be exemplified through two frameworks: DTCs, which are more common in treating mental health problems, and CTCs, which are generally applied when treating SUD [60]. In DTCs, psychodynamic principles are applied to enhance the patients' understanding of their current reaction patterns, considering their attachment style and their past experiences. CTCs, on the other hand, are more influenced by reward theory. Rewards and consequences are employed to change behaviours that are perceived to inhibit patients' recovery [60]. The variations in these approaches may be useful to illustrate differences in conventional perceptions about how the two conditions develop and progress. Such variations in conventional attitudes could have influenced the participants' reports on the RSA in this and previous studies.

Second, the way SUD and mental health problems are perceived and treated differs between cultures and countries. For instance, there are cultural variations in patterns of use, perceived harm, prevalence and acceptance of substances [62]. Additionally, differences in knowledge about causes, symptoms or the way the condition progresses may cause variations in how mental health problems are perceived in different cultures [63, 64]. Krendl and Pescosolido [64] illustrate this in their discussion. Based on their findings, the authors hypothesise that mental health conditions that are identified as treatable may generate a perception that low social function among people with mental health problems is their own choice. Cultural variations like these might have contributed to the issue with replicating the original five-factor structure shown in this and previous translations of the RSA.

Lastly, previous research has shown that there are major differences in how the notion of recovery is understood among clinicians and between services [65-67]. The clinicians in this study were not asked to outline their perceptions of recovery. However, variations in how recovery is understood may influence the implementation of recovery-orientation and which recovery measures are emphasised above others at the treatment facility.

Previous studies that have used RSA to examine recovery-orientation have been conducted in services both for people with mental health problems and for SUD. These studies have shown that the therapeutic orientation may influence the culture at the treatment facility and the clinicians' perceptions in various ways. Some studies showed that a higher degree of recoveryorientation at the treatment facility was associated with better work-related satisfaction [68], more positive attitudes towards patients [69] and less stigmatising attitudes among clinicians [42]. Other studies have shown positive associations between recovery-orientation and the treatment team of clinicians in terms of positive attitudes towards patients, better team climate and higher trust between team members [70,71]. We could not find any previous studies that solely used the RSA in specialised SUD services.

Considering this, the present study contributes to existing research by investigating RSA-N's potential as an instrument for assessing recovery-orientation exclusively in specialised SUD treatment. This contribution may induce a wider assessment of the degree to which SUD services and inpatient SUD treatment are recoveryoriented.

Some limitations of the current study should be considered. First, the data were obtained using a self-report questionnaire. Despite the advantages of using a selfreport questionnaire, the risk of social desirability bias is present. The questionnaire was completed anonymously by the participants, which reduced the risk of social desirability bias. Second, the response rate was acceptable but moderate (46\%). Also, larger facilities contributed with more responses compared to smaller facilities. However, participants from 50 out of the 54 eligible inpatient SUD treatment facilities in Norway responded to the questionnaire, which indicates that the data comprised a broad representation of the target population. Additionally, the sample size provided the opportunity to modify RSA-N and test the validity of the alternative model in two separate subsamples of the original sample. Lastly, the CFI value was close to but did not exceed 0.90 when testing the alternative model with the data from subsample 2 . The CFI could be improved by letting the error terms of several items to covary. However, this was not done due to the limitations of such an approach [50]. Additionally, the RMSEA values obtained for the alternative three-factor model suggested a good fit. Similarly, the alpha coefficients indicated satisfactory internal consistency for the three-factor RSA-N solution.

\section{Conclusions}

Since RSA was introduced in 2005, the instrument has been extensively used to assess the degree to which mental health services are recovery-oriented. However, RSA has hardly been used to assess recovery-orientation in SUD services and inpatient SUD treatment. In the current study, an alternative three-factor structure of the Norwegian translation - RSA-N - has obtained an acceptable fit and good internal consistency in a sample of clinicians working in inpatient SUD treatment facilities. The results are primarily supported by findings from earlier investigations of the instrument's psychometric properties $[11,13,14,19,58]$ and correspond with the results of previous examinations of its internal consistency [11-13, 18]. People with SUD are often 
stigmatised and discriminated in the broader society. Recovery-oriented measures have been known to reduce stigma and discrimination; therefore, it is significant that SUD services evaluate to what extent their practice is recovery-oriented. Knowledge about the degree to which SUD services are recovery-oriented, as well as knowledge about factors facilitating recovery-orientation in SUD services, may be important contributions in stigma prevention and in establishing an SUD treatment environment that fosters change and development of inpatients. The present study's findings imply RSA-N's potential as an instrument to assess recovery-orientation in inpatient SUD treatment.

\begin{abstract}
Abbreviations
CTC: Hierarchical Therapeutic Community; CFA: Confirmatory factor analysis; CFI: Comparative fit index; DTC: Democratic Therapeutic Community; NGO: Non-governmental organisations; RMSEA: Root mean square error of approximation; PCA: Principal component analysis; RSA: Recovery SelfAssessment; RSA-N: Recovery Self-Assessment - Norwegian; SUD: Substance use disorder
\end{abstract}

\section{Supplementary Information}

The online version contains supplementary material available at https://doi. org/10.1186/s13011-021-00363-0.

Additional file 1. Recovery Self-Assessment - Norwegian (RSA-N).

\section{Acknowledgments}

The authors would like to thank the participants for their contribution and the participating agencies for the recruitment to this study.

\section{Authors' contributions}

All three authors (DAJ, AØG and TN) contributed to the planning, interpretation of the results and writing the paper. DAJ collected the data and performed the analyses. The author(s) read and approved the final manuscript.

\section{Funding}

This study is part of a PhD degree which is funded by Blue Cross East in Norway. Blue Cross East took no other part in the research project or the included studies.

\section{Availability of data and materials}

The datasets generated and/or analysed during the current study are not publicly available due to ethical restrictions but are available from the corresponding author on reasonable request.

\section{Declarations}

\section{Ethics approval and consent to participate}

The study protocol was reviewed by the Norwegian Ethics Committee for Medical Research (REK) (reference number: 2017/1531) and then approved by the Norwegian Centre for Research Data (NSD; reference number 883511). The participants received written information and gave written consent.

\section{Consent for publication}

The participants gave their consent to publication by answering the first item in the questionnaire: "I give my consent to participate in the study and to my answers being stored in Sensitive Data Services (TSD) and used for the purpose of research."

\section{Competing interests}

The authors declare that they have no competing interests.

\section{Author details}

${ }^{1}$ Blue Cross East, Oslo, Norway. ${ }^{2}$ Department of Social Work, Child Welfare and Social Policy, OsloMet - Oslo Metropolitan University, Oslo, Norway. ${ }^{3}$ Department of Psychology, Norwegian University of Science and Technology (NTNU), Trondheim, Norway. ${ }^{4}$ St. Olavs Hospital, Clinic of Substance Use and Addiction Medicine, Trondheim, Norway.

Accepted: 9 March 2021

Published online: 19 March 2021

\section{References}

1. Meehan TJ, King RJ, Beavis PH, Robinson JD. Recovery-based practice: do we know what we mean or mean what we know? Aust N Z J Psychiatry. 2008;42(3):177-82. https://doi.org/10.1080/00048670701827234.

2. Davidson L, Tondora J, Lawless MS, O'Connell MJ, Rowe M. A practical guide to recovery-oriented practice: tools for transforming mental health care. New York: Oxford University Press; 2009.

3. Williams J, Leamy M, Bird V, Harding C, Larsen J, Le Boutillier C, Oades L, Slade M. Measures of the recovery orientation of mental health services: systematic review. Soc Psychiatry Psychiatr Epidemiol. 2012;47(11):1827-35. https://doi.org/10.1007/s00127-012-0484-y.

4. Slade M, Leamy M, Bacon F, Janosik M, Le Boutillier C, Williams J, Bird V. International differences in understanding recovery: systematic review. Epidemiol Psychiatr Sci. 2012;21:1-12.

5. Laudet AB. The road to recovery: where are we going and how do we get there? Empirically driven conclusions and future directions for service development and research. Subst Use Misuse. 2008;43(12-13):2001-20. https://doi.org/10.1080/10826080802293459.

6. Leamy M, Bird V, Le Boutillier C, Williams J, Slade M. Conceptual framework for personal recovery in mental health: systematic review and narrative synthesis. Br J Psychiatry. 2011;199(6):445-52. https://doi.org/10.1192/bjp. bp. 110.083733 .

7. Laudet $A B$, Humphreys $K$. Promoting recovery in an evolving policy context: what do we know and what do we need to know about recovery support services? J Subst Abus Treat. 2013;45(1):126-33. https://doi.org/10.1016/j.jsa t.2013.01.009.

8. Whitley R, Drake RE. Recovery: a dimensional approach. Psychiatr Serv. 2010; 61(12):1248-50. https://doi.org/10.1176/ps.2010.61.12.1248.

9. Burgess P, Pirkis J, Coombs T, Rosen A. Assessing the value of existing recovery measures for routine use in Australian mental health services. Austr N Z J Psychiatry. 2011;45(4):267-80. https://doi.org/10.3109/00048674.2010. 549996.

10. O'Connell M, Tondora J, Croog G, Evans A, Davidson L. From rhetoric to routine: assessing perceptions of recovery-oriented practices in a state mental health and addiction system. Psychiatr Rehabil J. 2005;28(4):378-86. https://doi.org/10.2975/28.2005.378.386.

11. Chao J, Siu AMH, Leung O, Lo A, Chu MZ, Lee WK, Auw C, Lee V, Chien CW. Chinese version of the recovery self-assessment scale: psychometric evidence from Rasch analysis and reliability estimates. J Ment Health. 2019; 28(2):206-12. https://doi.org/10.1080/09638237.2018.1521931.

12. Rosenberg D, Svedberg $P$, Schon UK. Establishing a recovery orientation in mental health services: evaluating the recovery self-assessment (RSA) in a Swedish context. Psychiatr Rehabil J. 2015;38(4):328-35. https://doi.org/10.1 037/prj0000150.

13. Tan KA, Fernandez A. Examining the reliability and factor structure of the Malay version of the recovery self-assessment in a sample of individuals with schizophrenia. Psychiatr Rehabil J. 2018;41(4):336-40. https://doi.org/1 $0.1037 /$ prj0000308.

14. Ye S, Pan J-Y, Wong DFK, Bola JR. Cross-validation of mental health recovery measures in a Hong Kong Chinese sample. Res Soc Work Pract. 2013;23(3): 311-25. https://doi.org/10.1177/1049731512471861.

15. Ricci EC, Leal E, La-Rotta EIG, Onocko-Campos R, O'Connell M. Cross-cultural adaptation of the recovery self-assessment instrument (RSA-R) person in recovery version to Brazilian Portuguese (Pt/Br). J Public Ment Health. 2020; 19(4):333-47. https://doi.org/10.1108/JPMH-02-2020-0008.

16. Zuaboni G, Kieser LD, Kozel B, Glavanovits K, Utschakowski J, Behrens J. Recovery self assessment: translation and cultural adoption of a recoveryoriented assessment instrument. Pflege. 2015;28(4):233-43. https://doi.org/1 0.1024/1012-5302/a000436.

17. Zuaboni G. Recovery-orientierte psychiatrische Akutpflege - Ergebnisse einer kontrollierten Interventionsstudie in zwei psychiatrischen Kliniken: Doctoral 
Thesis. Martin-Luther-Universität Halle-Wittenberg; 2017. http://dx.doi.org/1 $0.25673 / 2086$

18. Salyers MP, Tsai J, Stultz TA. Measuring recovery orientation in a hospital setting. Psychiatr Rehabil J. 2007;31(2):131-7. https://doi.org/10.2975/31.2.2 007.131.137.

19. Thege BK, Ham E, Ball LC. A factor analytic investigation of the person-inrecovery and provider versions of the revised recovery self-assessment (RSAR). Eval Health Prof. 2017;40(4):505-16. https://doi.org/10.1177/016327871 6674247.

20. Kraus SW, Stein CH. Recovery-oriented services for individuals with mental illness and case managers' experience of professional burnout. Community Ment Health J. 2013;49(1):7-13. https://doi.org/10.1007/s10597-012-9505-2.

21. Osborn LA, Stein CH. Mental health care Providers' views of their work with consumers and their reports of recovery-orientation, job satisfaction, and personal growth. Community Ment Health J. 2016;52(7):757-66. https://doi. org/10.1007/s10597-015-9927-8.

22. Fleury MJ, Sabetti J, Grenier G, Bamvita JM, Vallee C, Cao ZR. Work-related variables associated with perceptions of recovery-orientec care among. Quebec mental health professionals. BJPsych Open. 2018:4(6):478-85. https://doi.org/10.1192/bjo.2018.66.

23. Leddy-Stacy M, Stefanovics E, Rosenheck R. Veteran and clinician perceptions of recovery and stigma at a veterans affairs medical center. Psychiatr Rehabil J. 2016;39(2):105-11. https://doi.org/10.1037/prj0000174.

24. Kidd SA, George L, O'Connell M, Sylvestre J, Kirkpatrick H, Browne G, Odueyungbo AO, Davidson L. Recovery-oriented service provision and clinical outcomes in assertive community treatment. Psychiatr Rehabil J. 2011;34(3):194-201. https://doi.org/10.2975/34.3.2011.194.201.

25. Best D. Strength, support, setbacks and solutions: the developmental pathway to addiction recovery, vol. 152. Hove: Pavilion; 2014.

26. Camilleri AC, Cacciola JS, Jenson MR. Comparison of two ASI-based standardized patient placement approaches. J Addict Dis. 2012;31(2):118-29. https://doi.org/10.1080/10550887.2012.665727.

27. Lopez-Goni JJ, Fernandez-Montalvo J, Arteaga A, Esarte S. Searching objective criteria for patient assignment in addiction treatment. J Subst Abus Treat. 2017;76:28-35. https://doi.org/10.1016/j.jsat.2017.02.014.

28. Wakeman SE, Metlay JP, Chang Y, Herman GE, Rigotti NA. Inpatient addiction consultation for hospitalized patients increases post-discharge abstinence and reduces addiction severity. J Gen Intern Med. 2017;32(8): 909-16. https://doi.org/10.1007/s11606-017-4077-z.

29. Johnson R, Haigh R. Social psychiatry and social policy for the 21st century - new concepts for new needs: the 'psychologically-informed environment'. Ment Health Soc Incl. 2010;14(4):30-5. https://doi.org/10. 5042/mhsi.2010.0620

30. Haigh R, Harrison T, Johnson R, Paget S, Williams S. Psychologically informed environments and the "enabling environments" initiative. Housing Care Supp. 2012;15(1):34-42. https://doi.org/10.1108/14608791211238412.

31. Johnson R, Haigh R. Social psychiatry and social policy for the 21st century: new concepts for new needs - the 'Enabling Environments' initiative. Ment Health Soc Incl. 2011;15(1):17-23. https://doi.org/10.5042/mhsi.2011.0054

32. Pettersen $H$, Landheim A, Skeie I, Biong S, Brodahl M, Oute J, Davidson L. How social relationships influence substance use disorder recovery: a collaborative narrative study. Subst Abus. 2019;13:1-8.

33. Bahl NKH, Nafstad HE, Blakar RM, Landheim AS, Brodahl M. Multiple senses of community and recovery processes. A pilot study for a national evaluation of the experiences of persons with substance use problems receiving help and services from Norwegian municipalities. J Commun Psychol. 2019;47(6):1-20.

34. Eslami AA, Norozi E, Hajihosseini M, Ramazani AA, Miri MR. Social cognitive theory as a theoretical framework to predict sustained abstinence 6 months after substance use treatment. J Subst Abus. 2018;23(3):300-6.

35. Andersson HW, Otterholt E, Gråwe RW. Patient satisfaction with treatments and outcomes in residential addiction institutions. Nordic Stud Alcohol Drugs. 2017;34(5):375-84. https://doi.org/10.1177/1455072517718456.

36. Johannessen DA, Nordfjærn T, Geirdal AØ. Substance use disorder patients expectations on transition from treatment to post-discharge period. Nordic Stud Alcohol Drugs. 2020;37(3):208-26. https://doi.org/10.1177/145507252 0910551.

37. Bergman BG, Hoeppner BB, Nelson LM, Slaymaker V, Kelly JF. The effects of continuing care on emerging adult outcomes following residential addiction treatment. Drug Alcohol Depend. 2015;153:207-14. https://doi. org/10.1016/j.drugalcdep.2015.05.017.
38. Manuel JI, Yuan Y, Herman DB, Svikis DS, Nichols O, Palmer E, Deren S Barriers and facilitators to successful transition from long-term residential substance abuse treatment. J Subst Abus Treat. 2017;74:16-22. https://doi. org/10.1016/j.jsat.2016.12.001.

39. Barry CL, McGinty EE, Pescosolido BA, Goldman HH. Stigma, discrimination, treatment effectiveness, and policy: public views about drug addiction and mental illness. Psychiatr Serv. 2014;65(10):1269-72. https://doi.org/10.1176/a ppi.ps.201400140.

40. Kulesza M, Larimer ME, Rao D. Substance use related stigma: what we know and the way forward. J Addict Behav Ther Rehabil. 2013;2(2):27.

41. van Boekel LC, Brouwers EPM, van Weeghel J, Garretsen HFL. Stigma among health professionals towards patients with substance use disorders and its consequences for healthcare delivery: systematic review. Drug Alcohol Depend. 2013;131(1-2):23-35. https://doi.org/10.1 016/j.drugalcdep.2013.02.018.

42. Stacy MA, Rosenheck R. The association of recovery orientation and stigmatizing beliefs. J Ment Health. 2019;28(3):276-81. https://doi.org/10.1 080/09638237.2017.1417573.

43. Kelly JF, Westerhoff CM. Does it matter how we refer to individuals with substance-related conditions? A randomized study of two commonly used terms. Int J Drug Policy. 2010;21(3):202-7. https://doi.org/10.1016/j.drugpo.2 009.10.010.

44. Ashford RD, Brown AM, Curtis B. "Abusing addiction": our language still Isn't good enough. Alcohol Treat Q. 2019;37(2):257-72. https://doi.org/10.1080/ 07347324.2018.1513777.

45. Wild D, Grove A, Martin M, Eremenco S, McElroy S, Verjee-Lorenz A, Erikson P. Principles of good practice for the translation and cultural adaptation process for patient-reported outcomes (PRO) measures: report of the ISPOR task force for translation and cultural adaptation. Value Health. 2005;8(2):94104. https://doi.org/10.1111/j.1524-4733.2005.04054.x.

46. Campbell-Orde T, Chamberlin J, Carpenter J, Stephen LH. Measuring the Promise: A Compendium of Recovery Measures; 2005.

47. Dale-Perera A. Recovery, reintegration, abstinence, harm reduction: The role of different goals within drug treatment in the European context; 2017.

48. Sumnall H, Brotherhood A. Social reintegration and employment: Evidence and interventions for drug users in treatment; 2012.

49. UNODC, WHO. International standards for the treatment of drug use disorders; 2016

50. Brown TA. Confirmatory factor analysis for applied research, second edition. New York: Guilford Publications; 2015.

51. Mehmetoglu M, Jakobsen TG. Applied statistics using stata: a guide for the social sciences. Los Angeles: SAGE Publications; 2016.

52. Kline RB. Principles and practice of structural equation modeling. 3rd ed. New York: Guilford Press; 2011.

53. Hu L-t, Bentler PM. Cutoff criteria for fit indexes in covariance structure analysis: conventional criteria versus new alternatives. Struct Equ Model. 1999:6(1):1-55. https://doi.org/10.1080/10705519909540118.

54. Christophersen K-A. Databehandling og statistisk analyse med SPSS [data processing and statistical analysis with SPSS]. 4th ed. Oslo: Unipub; 2009.

55. Schumacker RE. Learning statistics using R. London: SAGE Publications, Inc; 2015. https://doi.org/10.4135/9781506300160.

56. The jamovi project. jamovi. Version 1.2.27. jamovi; 2020. https://doi.org/10.11 86/s13011-021-00363-0.

57. Vogt WP. Dictionary of statistics \& methodology : a nontechnical guide for the social sciences, vol. 1. 3rd ed. Thousand Oaks: SAGE; 2005. https://doi. org/10.4135/9781412983907.

58. Lodge AC, Kuhn W, Earley J, Manser SS. Initial development of the recoveryoriented services assessment: a collaboration with peer-provider consultants. Psychiatr Rehabil J. 2018;41(2):92-102. https://doi.org/10.1037/prj0000296.

59. Soyez V, Melnick G, De Leon G, Broekaert E, Goethals I, Leon GD. Essential elements of treatment: a comparative study between European and American therapeutic communities for addiction. Journal. 2011:46(8):102331. https://doi.org/10.3109/10826084.2010.544358.

60. Akerman G. Communal living as the agent of change. In: DLL P, Day A, Hollin CR, editors. The Wiley International Handbook of Correctional Psychology; 2019. p. 590-602.

61. Brown AM, Ashford RD. Recovery-informed theory: situating the subjective in the science of substance use disorder recovery. J Recov Sci. 2019;1(3):115. https://doi.org/10.31886/jors.13.2019.38.

62. Room R, Janca A, Bennett LA, Schmidt L, Sartorius N. WHO cross-cultural applicability research on diagnosis and assessment of substance use 
disorders: an overview of methods and selected results. Addiction. 1996; 91(2):199-220, discussion 221-130. https://doi.org/10.1111/j.1360-0443.1996. tb03176.x.

63. Choudhry FR, Mani V, Ming LC, Khan TM. Beliefs and perception about mental health issues: a meta-synthesis. Neuropsychiatr Dis Treat. 2016;12: 2807-18. https://doi.org/10.2147/NDT.S111543.

64. Krendl AC, Pescosolido BA. Countries and cultural differences in the stigma of mental illness: the east-west divide. J Cross-Cult Psychol. 2020;51(2):14967. https://doi.org/10.1177/0022022119901297.

65. Coffey M, Hannigan B, Barlow S, Cartwright M, Cohen R, Faulkner A, Jones A, Simpson A. Recovery-focused mental health care planning and coordination in acute inpatient mental health settings: a cross national comparative mixed methods study. BMC Psychiatry. 2019;19(1):115. https:// doi.org/10.1186/s12888-019-2094-7.

66. Le Boutillier C, Chevalier A, Lawrence V, Leamy M, Bird VJ, Macpherson R, Williams J, Slade M. Staff understanding of recovery-orientated mental health practice: a systematic review and narrative synthesis. Implement Sci. 2015;10(1):87. https://doi.org/10.1186/s13012-015-0275-4.

67. Cutcliffe JR, Santos JC, Kozel B, Taylor P, Lees D. Raiders of the lost art: a review of published evaluations of inpatient mental health care experiences emanating from the United Kingdom, Portugal, Canada, Switzerland, Germany and Australia. Int J Ment Health Nurs. 2015;24(5):375-85. https:// doi.org/10.1111/inm.12159

68. Rabenschlag F, Konrad A, Rueegg S, Jaeger M. A recovery-oriented approach for an acute psychiatric Ward: is it feasible and how does it affect staff satisfaction? Psychiatry Q. 2014;85(2):225-39. https://doi.org/10.1007/ s11126-013-9285-z

69. Hornik-Lurie T, Shalev A, Haknazar L, Epstein PG, Ziedenberg-Rehav L, Moran GS. Implementing recovery-oriented interventions with staff in a psychiatric hospital: a mixed-methods study. J Psychiatr Ment Health Nurs. 2018;25(910):569-81. https://doi.org/10.1111/jpm.12502.

70. Leamy M, Clarke E, Le Boutillier C, Bird V, Choudhury R, MacPherson R, Pesola F, Sabas K, Williams J, Williams P, et al. Recovery practice in community mental health teams: national survey. Br J Psychiatry. 2016; 209(4):342-8.

71. Fleury MJ, Sabetti J, Bamvita JM. Modeling relationships involving perceived recovery orientation of mental health teams among Quebec mental health professionals. J Behav Health Serv Res. 2019;46(3):434-49. https://doi.org/1 0.1007/s11414-018-9591-1.

\section{Publisher's Note}

Springer Nature remains neutral with regard to jurisdictional claims in published maps and institutional affiliations.

Ready to submit your research? Choose BMC and benefit from:

- fast, convenient online submission

- thorough peer review by experienced researchers in your field

- rapid publication on acceptance

- support for research data, including large and complex data types

- gold Open Access which fosters wider collaboration and increased citations

- maximum visibility for your research: over $100 \mathrm{M}$ website views per year

At $\mathrm{BMC}$, research is always in progress.

Learn more biomedcentral.com/submissions 